\title{
Design of High-speed Sampling System in Pulse Laser Application
}

\author{
Jian Liu ${ }^{1}$, Ming-ai Lv², Jiang Wang ${ }^{2}$ \\ ${ }^{1}$ The Engineering \& Technical College, Chengdu University of Technology, Leshan, China \\ ${ }^{2}$ Southwest Institute of Technical Physics, Chengdu, China \\ Email: emailofliujian@126.com
}

Received 2013

\begin{abstract}
In measurement system by means of pulse laser, such as plasma measuring, laser ranging, the amplitude of echoed laser wave is very weak and difficult to detect by traditional analog electronic technology. A digital high speed data acquisition and processing system was designed to meet the accuracy requirement. It adopted high speed AD chip and advantage FPGA chip as core unit. Experiment results have verified this system can reach to $1 \mathrm{GHz}$ sample rate and can catch weak echo wave effectively and the measuring accuracy is improved markedly.
\end{abstract}

Keywords: Pulse Laser; Echo Wave Detecting; High Speed AD; FPGA

\section{Introduction}

In many engineering application, such as plasma characteristics measuring, laser ranging, remote dust concentration measuring, and so on, the pulse laser technology were adopted widely[1-4]. As the precision requirement is higher, the pulse width is narrower, and the echo wave energy received by the detector is lower. Under this circumstance, even the received signal has been amplified highly, it is very weakly still.

In recently application system of pulse laser, the pulse could be compressed to narrower than $5 \mathrm{~ns}$. But because of the spread effect of plasma or atmosphere, the pulse would be broadened about to $5-15$ ns. When the breadth became narrower and plasma density became higher, the amplitude of the echo pulse signal received by measuring system became weaker rapidly, and traditional analog electronic system couldn't catch it. Figure 1 shows a common receiving wave picture on oscilloscope of these cases.

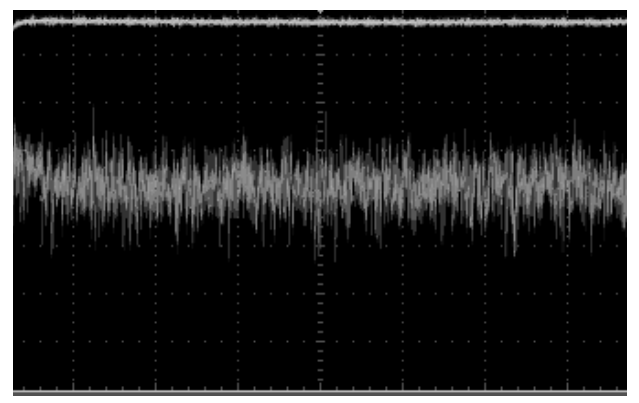

Figure 1. Typical received echo wave pulse.
To deal with such cases, a pulse signal detecting and processing system was designed. It adopted high speed $\mathrm{AD}$ device and the fast data processing ability of FPGA. This system can well work under condition of low signal to noise ratio (SNR), narrow pulse width, little echo wave amplitude. In the system, the AD chip ADC08D1000 which work in $1 \mathrm{GHz}$ sampling rate, and the EP2S60 FPGA chip, are applied as core components.

\section{System Design}

\subsection{High Speed Sampling Sub-system}

To realize digital sampling of pulse signal, a AD subsystem was designed firstly, and the sketch of its structure is shown in Figure 2. Sampling rate of the AD chip was controlled by external clock, and the output of sample data was in differential format to enhance capacity of resistant disturbance. In the same time, an accompany clock was outputted to lock the phase of the multiplexed output of sample data.

In order to improve the measuring accuracy, the width of pulse laser would be narrow as possible. In this system, the pulse is $5 \mathrm{~ns}$ width. It required the AD device was in very high sampling rate, and then ADC08D1000 was selected because it had two-channel sample function and

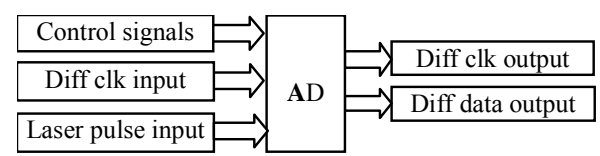

Figure 2. Sketch of high speed sampling subsystem. 
could work at $1 \mathrm{GHz}$ sampling rate. The IO list of ADC08D1000 was shown in Figure 3.

After AD chip was determined, the clock circuit which provided a stable $1 \mathrm{GHz}$ clock to the $\mathrm{AD}$ chip must be considered. In the system, it was separated and consisted of outer crystal oscillator, phase lock loop, voltage controlled oscillator, etc. Its schematic is shown in Figure 4, and signals PLL_SCLK, PLL_SDATA, PLL_LE were produced by FPGA, and signal OSCin was produced by outer low-frequency oscillator.

By accepting the clock produced by the clock circuit and the sampling sequence controlling signal produced by a processor, the sample subsystem then work.

\subsection{High Speed Data Sink and Process Subsystem}

In order to cooperate with the speed of the sampling sub-system, data sink and process speed of the processor must match to the sample rate. Finally the Stratix II series FPGA chip EP2S60 was used in advantage of its large inner resource, high data handle speed. This processor can work at $550 \mathrm{MHz}$ clock frequency and has 12 PLLs unit, a huge amount memory larger than $9 \mathrm{M}$ bits, and high speed differential IO ports with dynamic phase aligner (DPA) function and can support $1040 \mathrm{MHz}$ data sink. These capabilities can satisfy the resource requirement of the system.

The FPGA chip would produce controlling signals including PLL_clk, PLL_data, PLL_le, to control the outer clock circuit, and made it output a $1 \mathrm{GHz}$ clock signal to AD chip. The logical module in FPGA was illuminated by Figure 5.

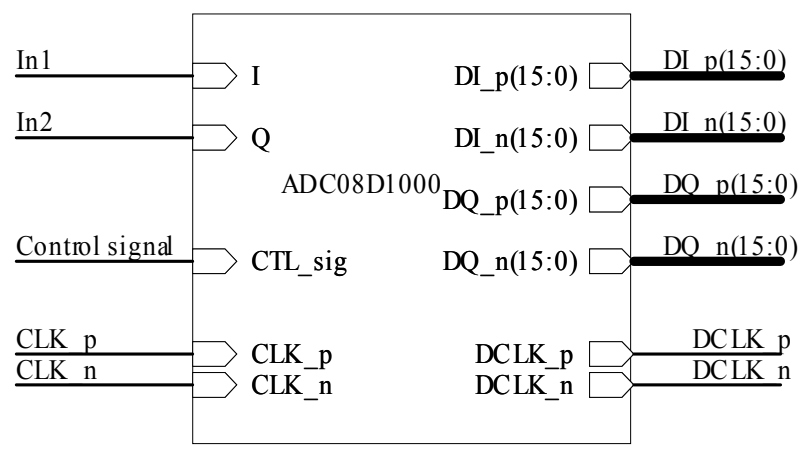

Figure 3. High speed AD's structure.

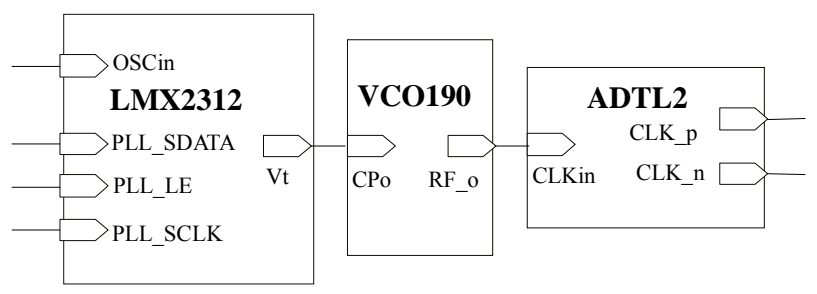

Figure 4. Sketch of circuit controlling AD's clock.

\subsection{Integration of the Completed System}

By integrated the two subsystem, the completed system was gotten. Sketch of the whole system was shown in Figure 6.

From Figure 6, we can see that the most important part of the system is the logical unit in FPGA, which control the outer clock circuit, AD component and data sink. This logical unit was designed as the functional flow diagram as showed in Figure 7.

As described in Figure 7, the system is firstly to configure the clock controlling signal and reset the dpa signal of LVDS port, and then wait for data sink writing signal (DSWS). After received DSWS, it begins to accept and store data, and then prepare these data to user.

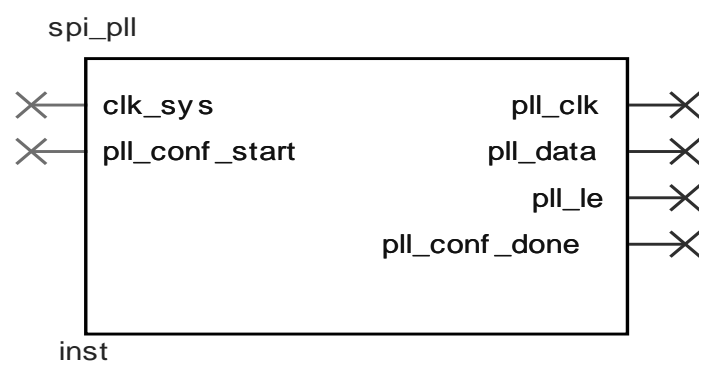

Figure 5. Logical controlling module in FPGA.

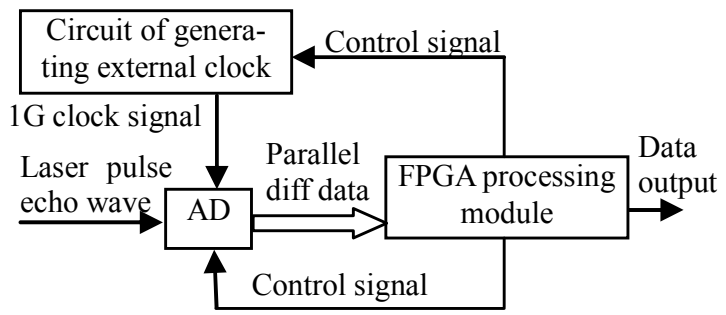

Figure 6. Sketch of integrated system.

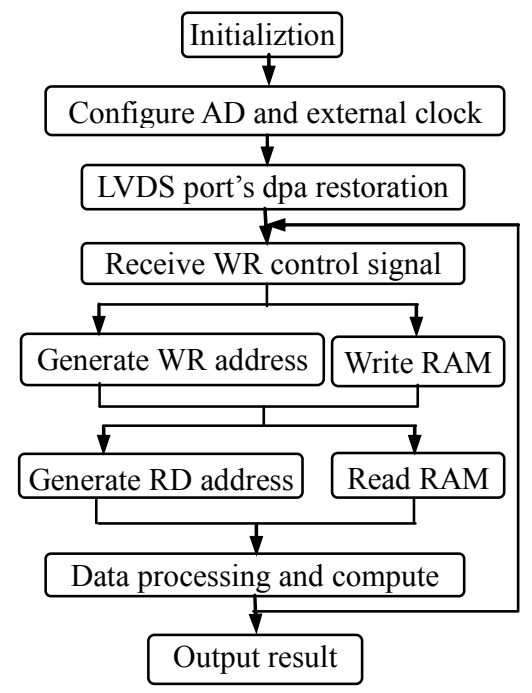

Figure 7. Functional flow of the program in FPGA. 
After the data was completely received, they were stored into double ports RAM firstly, and then read out by a lower lock frequency. Analyzing these data, combining the automatic threshold judgment, the echo wave signal of pulse laser could be find out accurately.

\section{Experimental Results and Analysis}

To testing the feasibility of this design, an integrated hardware system was made, and many experiments were done. Applied the system in distance ranging equipment, experimental results of four distance cases were obtained and listed in Table 1. In the table, Std means the standard distance number, Max and Min means the maximum and minimum number of ranging results, Aver means the average value, $\delta$ means the standard variance, and they are all in unit of meter.

Plotting one group experimental data in Figure 8, and the ordinate is the distance obtained by ranging, and the abscissa is the repeated times of ranging.

From Table 1 and Figure 8, it could be seen that the sampling system was well work. Because of its $1 \mathrm{GHz}$ high sampling rate, the distance ranging equipment achieved to $\pm 20 \mathrm{~mm}$ precision whether in short or in long distance ranging.

Table 1. Experimental results and error analysis.

\begin{tabular}{ccccc}
\hline Case & 1 & 2 & 3 & 4 \\
\hline Std & 0.492 & 19.844 & 249.591 & 1002.617 \\
Max & 0.503 & 19.843 & 249.606 & 1002.618 \\
Min & 0.489 & 19.830 & 249.588 & 1002.605 \\
Aver & 0.498 & 19.838 & 249.598 & 1002.613 \\
$\delta$ & 0.0120 & 0.0099 & 0.0127 & 0.0109 \\
\hline
\end{tabular}

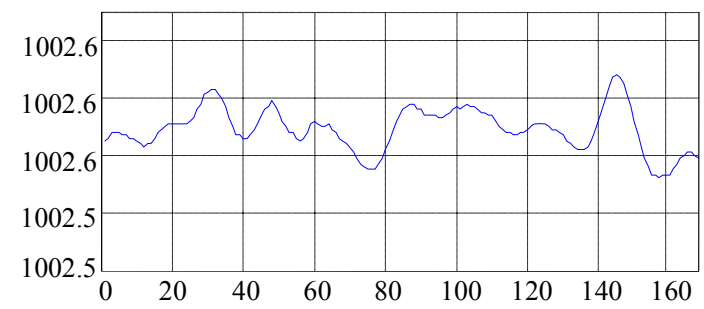

Figure 8. The plotting of experimental ranging data.

\section{Conclusions}

To solving the low SNR and narrow echo wave detcting problem in pulse laser measuring, adopted high speed AD chip and Stratix II series FPGA as the core units, a high speed digital sampling system was designed. The experimental results verified the design could improve the acquisition accuracy of the echo wave signal markedly.

\section{REFERENCES}

[1] Z. H. Sun, Y. W. Xia and J. D., "Temporal Measurement of Nanosecond Laser Pulse Based on Wave Clipping of Plasma," High Power Laser and Paticle Beams, Vol. 24, No. 8, 2012, pp. 1846-1850.

[2] Q. Deng, L. LI, M. Ma, et al., "High Precision Time Measurement and Its Application in Pulsed Laser Ranging," Electronic and Electro-optical Systems, No. 2, 2010, pp. 11-16.

[3] L. L. Zhang, J. T. Fu, Z. Y. Zhang, et al., "Application of FPGA in Long Distance Laser Ranging," LASER \& INFRARED, Vo1. 40, No. 3, 2010, pp. 287-289.

[4] Z. Q. Tong, "Design of Clock System in High Speed Data Acquisition," Journal of Harbin University Science and Technology, Vol. 12, No. 3, 2007, pp. 36-39. 